Bull. Austral. Math. Soc.

VOL. 48 (1993) [7-12]

\title{
INEQUALITIES WITH WEIGHTS FOR POWERS OF GENERALISED INVERSES
}

\author{
B. Mond and J.E. PEČarić
}

\begin{abstract}
Recently the authors extended to positive semi-definite matrices converses of matrix inequalities corresponding to ratios and differences of means and of Hölder's inequality. Here, further generalisations are given to the case of several vectors with appropriate weights.
\end{abstract}

\section{INTRODUCTION}

Generalisations of some well-known converses of means inequalities involving powers of generalised inverses were given in [3]. Here we give further generalisations in the case of several vectors.

Notation AND PREVIOUS RESULTS.

Given any matrix $A$ with complex entries, there exists a unique matrix $A^{+}$satisfying

$$
A A^{+} A=A, A^{+} A A^{+}=A^{+}, A A^{+}=\left(A A^{+}\right)^{*}, A^{+} A=\left(A^{+} A\right)^{*}
$$

where ${ }^{*}$ denotes conjugate transpose. $A^{+}$is called the (Moore-Penrose) generalised inverse of $A$.

Let $A$ be a positive semi-definite Hermitian matrix of order $n$ and rank $k$. Let those roots of $A$ which are strictly positive be ordered as $\lambda_{1} \geqslant \lambda_{2} \geqslant \ldots \geqslant \lambda_{k}>0$. For simplicity of notation, if $r<0$, we shall use $A^{(r)}$ for $\left(A^{+}\right)^{-r}$. Note that, with $r<0,\left(A^{+}\right)^{-r}=\left(A^{-r}\right)^{+}$. Also if $k=n, A^{+}=A^{-1}$ and $A^{(r)}=A^{r}$.

In our proofs we need the following result from [3].

Lemma 1. Let $r$ and $s$ be real numbers, $r<0, s>0$. Let $A$ be a positive semi-definite Hermitian matrix of order $n$ and rank $k$. Let those roots of $A$ which are strictly positive be ordered as $\lambda_{1} \geqslant \lambda_{2} \geqslant \ldots \geqslant \lambda_{k}>0, \lambda_{k}<\lambda_{1}$. Set

$$
a=\frac{\lambda_{1}^{r}-\lambda_{k}^{r}}{\lambda_{1}^{\prime}-\lambda_{k}^{s}}, \quad b=\frac{\lambda_{1}^{s} \lambda_{k}^{r}-\lambda_{1}^{r} \lambda_{k}^{s}}{\lambda_{1}^{*}-\lambda_{k}^{r}}
$$

Let $B$ be a Hermitian matrix that satisfies

Received 3rd July, 1992

Copyright Clearance Centre, Inc. Serial-fee code: 0004-9729/93 \$A2.00+0.00. 


$$
A^{-r} B A^{-r}=A^{-r}, A^{-r} B=B A^{-r}
$$

and has $n-k$ non-positive roots. Then for any $x$ such that $x^{*} x=1$

$$
x^{*} B x-a x^{*} A^{*} x-b \leqslant 0 .
$$

Strict equality holds if and only if $x=u_{1}+u_{k}$ where $A u_{1}=\lambda_{1} u_{1}$ and $A u_{k}=\lambda_{k} u_{k}$. Results for MEANS.

First we state a theorem that is equivalent to Lemma 1.

Theorem 1. Let $r, s, A$ and $B$ be defined as in Lemma 1. Further, let $w_{j}, j=$ $1, \ldots, m$ be positive numbers with $\sum_{j=1}^{m} w_{j}=1$ and let $x_{j}, j=1, \ldots, m$, be such that $x_{j}^{*} x_{j}=1, j=1, \ldots, m$. Then

$$
\sum_{j=1}^{m} w_{j} x_{j}^{*} B x_{j}-a \sum_{j=1}^{m} w_{j} x_{j}^{*} A^{\prime} x_{j}-b \leqslant 0 .
$$

Strict equality holds if and only if $x_{j}=u_{j 1}+u_{j k}$ where $A u_{j 1}=\lambda_{1} u_{j 1}$ and $A u_{j k}=$ $\lambda_{k} u_{j k}$.

Proof: By (2) we have

$$
x_{j}^{*} B x_{j}-a x_{j}^{*} A^{*} x_{j}-b \leqslant 0 .
$$

Multiplying this inequality by $\boldsymbol{w}_{j}$, and adding, we get (3).

TheOREM 2. Let $r, s, A, B, w_{j}, x_{j}, j=1, \ldots, m$ be as in Theorem 1 and $\gamma=$ $\lambda_{1} / \lambda_{k}$. If $\sum_{j=1}^{m} w_{j} x_{j}^{*} B x_{j} \geqslant 0$, then

$$
\left(\sum_{j=1}^{m} w_{j} x_{j}^{*} A^{\prime} x_{j}\right)^{1 / \bullet}\left(\sum_{j=1}^{m} w_{j} x_{j}^{*} B x_{j}\right)^{-1 / r} \leqslant \Omega
$$

where

$$
\Omega=\left\{\frac{r\left(\gamma^{*}-\gamma^{r}\right)}{(s-r)\left(\gamma^{r}-1\right)}\right\}^{1 / \bullet}\left\{\frac{s\left(\gamma^{r}-\gamma^{*}\right)}{(r-s)\left(\gamma^{s}-1\right)}\right\}^{-1 / r} .
$$

Strict equality holds if and only if $x_{j}=u_{j 1}+u_{j k}$ where $A u_{j 1}=\lambda_{1} u_{j 1}$ and $A u_{j k}=\lambda_{k} u_{j k}$ and

$$
\sum_{j=1}^{m} w_{j} u_{j 1}^{*} u_{j 1}=\left[r /\left(\gamma^{r}-1\right)-s /\left(\gamma^{\bullet}-1\right)\right] /(s-r)
$$


Proof: By Theorem 1, with $a$ and $b$ defined by (1),

$$
\begin{gathered}
\left(\sum_{j=1}^{m} w_{j} x_{j}^{*} A^{*} x_{j}\right)^{1 / \bullet}\left(\sum_{j=1}^{m} w_{j} x_{j}^{*} B x_{j}\right)^{-1 / r} \\
\leqslant\left(\sum_{j=1}^{m} w_{j} x_{j}^{*} A^{s} x_{j}\right)^{1 / \bullet}\left[a \sum_{j=1}^{m} w_{j} x_{j}^{*} A^{\prime} x_{j}+b\right]^{-1 / r} \equiv g\left(\sum_{j=1}^{m} w_{j} x_{j}^{*} A^{s} x_{j}\right)
\end{gathered}
$$

where $g(t)=t^{1 / \bullet}[a t+b]^{-1 / r}$. Here $g(t)$ is easily seen to have a maximum at $t=$ $r b[a(s-r)]^{-1}$ which gives the upper bound of (6).

Strict equality holds if and only if it holds in (4) at

$$
\sum_{j=1}^{m} w_{j} x_{j}^{*} A^{*} x_{j}=r b[a(s-r)]^{-1} .
$$

Given the condition for strict equality in (3), strict equality in (7) is easily seen to hold if and only if it holds in (6).

Note that the above proof is similar to the proof of Theorem 2 from [3]. In a similar manner to the proof of Theorem 3 in [3], we can prove the following.

Theorem 3. Let $r, s, A, B, a, b, w_{i}, x_{i}, i=1, \ldots, m$ be defined as in Theorem 1. If $\sum_{i=1}^{m} w_{i} x_{i}^{*} B x_{i}>0$, then

$$
\left(\sum_{i=1}^{m} w_{i} x_{i}^{*} A^{\prime} x_{i}\right)^{1 / \bullet}-\left(\sum_{i=1}^{m} w_{i} x_{i}^{*} B x_{i}\right)^{1 / r} \leqslant \triangle
$$

where

$$
\Delta=\left[\theta \lambda_{1}^{s}+(1-\theta) \lambda_{k}^{s}\right]^{1 / \Delta}-\left[\theta \lambda_{1}^{r}+(1-\theta) \lambda_{k}^{r}\right]^{1 / r} .
$$

$\theta$ is defined as follows. Let

$$
h(y)=y^{1 / \bullet}-(a y+b)^{1 / r} .
$$

Let $J$ denote the open interval joining $\lambda_{k}^{\dot{*}}$ to $\lambda_{i}^{*}$. Let $\bar{J}$ be the closure of $J$. There is a unique $y^{*} \in \bar{J}$ where $h(y)$ attains its maximum in $\bar{J}$. This $y^{*}$ lies in $J$. Set

$$
\theta=\frac{y^{*}-\lambda_{k}^{s}}{\lambda_{1}^{s}-\lambda_{k}^{s}}
$$

Strict equality holds if and only if $x_{j}=u_{j 1}+u_{j k}$ where $A u_{j 1}=\lambda_{1} u_{j 1}, A u_{j k}=\lambda_{k} u_{j k}$ and $\sum_{j=1}^{m} w_{j} u_{j 1}^{*} u_{j 1}=\theta$. If $s \geqslant 1$, then $y^{*}$ is the unique solution of $h^{\prime}(y)=0$ in $J$. 
Since $A^{(r)}$ satisfies the conditions for $B$ in Lemma $1,(3),(4)$ and (8) yield

$$
\sum_{j=1}^{m} w_{j} x_{j}^{*} A^{(r)} x_{j}-a \sum_{j=1}^{m} w_{j} x_{j}^{*} A^{*} x_{j}-b \leqslant 0,
$$

where $a$ and $b$ are defined by (1);

$$
\left(\sum_{j=1}^{m} w_{j} x_{j}^{*} A^{*} x_{j}\right)^{1 / s}\left(\sum_{j=1}^{m} w_{j} x_{j}^{*} A^{(r)} x_{j}\right)^{-1 / r} \leqslant \Omega,
$$

where $\Omega$ is given by (5), and

$$
\left(\sum_{j=1}^{m} w_{j} x_{j}^{*} A^{s} x_{j}\right)^{1 / s}-\left(\sum_{j=1}^{m} w_{j} x_{j}^{*} A^{(r)} x_{j}\right)^{1 / r} \leqslant \triangle,
$$

where $\Delta$ is given by (9).

REMARK: (8) required $\sum_{j=1}^{m} w_{j} x_{j}^{*} B x_{j}>0$. Since $\sum_{j=1}^{m} w_{j} x_{j}^{*} A^{(r)} x_{j} \geqslant 0$ for all $x_{j}$, we may regard (12) as being satisfied with strict inequality when $\sum_{j=1}^{m} w_{j} x_{j}^{*} A^{(r)} x_{j}=0$. (A similar remark will be applicable to $\sum_{j=1}^{m} w_{j} x_{j}^{*} A^{+} x_{j}=0$ in equation (18).)

\section{Special Cases}

Our results generalise a number of well-known inequalities. Thus, if $k=n$, that is, $A$ is positive definite, $A^{+}=A^{-1}$ and $B=A^{(r)}=A^{r}$, and our results yield well-known inequalities for positive definite matrices.

Interesting special cases are, for $r=-1, s=1$,

$$
\begin{aligned}
& \sum_{j=1}^{m} w_{j} x_{j}^{*} A x_{j}+\lambda_{1} \lambda_{k} \sum_{j=1}^{n} w_{j} x_{j}^{*} B x_{j} \leqslant \lambda_{1}+\lambda_{k} \\
&\left(\sum_{j=1}^{m} w_{j} x_{j}^{*} A x_{j}\right)\left(\sum_{j=1}^{m} w_{j} x_{j}^{*} B x_{j}\right) \leqslant\left(\lambda_{1}+\lambda_{k}\right)^{2} /\left(4 \lambda_{1} \lambda_{k}\right) ; \\
&\left(\sum_{j=1}^{m} w_{j} x_{j}^{*} A x_{j}\right)-\left(\sum_{j=1}^{m} w_{j} x_{j}^{*} B x_{j}\right)^{-1} \leqslant\left(\sqrt{\lambda_{1}}-\sqrt{\lambda_{k}}\right)^{2} .
\end{aligned}
$$

REMARK: In our results for (4) we have the condition

$$
\sum_{j=1}^{m} w_{j} x_{j}^{*} B x_{j} \geqslant 0 .
$$


It is obvious that (14) is valid without this condition and thus is a generalisation of a result from [2].

Note that (14) as well as Theorem 3 are generalisations of an inequality of Ky Fan [1].

Of special interest is the case where $B$ is the generalised inverse of $A$. In this case (13), (14) and (15) become

$$
\begin{aligned}
& \sum_{j=1}^{m} w_{j} x_{j}^{*} A x_{j}+\lambda_{1} \lambda_{k} \sum_{j=1}^{m} w_{j} x_{j}^{*} A^{+} x_{j} \leqslant \lambda_{1}+\lambda_{k} ; \\
&\left(\sum_{j=1}^{m} w_{j} x_{j}^{*} A x_{j}\right)\left(\sum_{j=1}^{m} w_{j} x_{j}^{*} A^{+} x_{j}\right) \leqslant\left(\lambda_{1}+\lambda_{k}\right)^{2} / 4 \lambda_{1} \lambda_{2} ; \\
&\left(\sum_{j=1}^{m} w_{j} x_{j}^{*} A x_{j}\right)-\left(\sum_{j=1}^{n} w_{j} x_{j}^{*} A^{+} x_{j}\right)^{-1} \leqslant\left(\sqrt{\lambda_{1}}-\sqrt{\lambda_{k}}\right)^{2} .
\end{aligned}
$$

As noted (18) may be regarded as being satisfied with a strict inequality when

$$
\sum_{j=1}^{m} w_{j} x_{j}^{*} A^{+} x_{j}=0
$$

HÖLDER'S INEQUALITY.

The following result proved in [3] will be used to establish a generalisation of the converse of a matrix form of Hölder's inequality.

Lemma 2. [3] Let $r, s, p$ and $q$ be real numbers such that $r>0, s>0,0<p<1$ and $p^{-1}+q^{-1}=1, \alpha=p s-q r$. Let $A$ be defined as in Lemma 1 and let $C$ be a Hermitian matrix that satisfies

$$
A^{-r q} C A^{-r q}=A^{-r q}, A^{-r q} C=C A^{-r q}
$$

and has $n-k$ non-positive roots. Then for any $x$, we have

$$
\lambda_{k}^{-\alpha / q}\left(\gamma^{\alpha / p}-1\right) x^{*} A^{s p} x+\lambda_{1}^{\alpha / p}\left(\gamma^{\alpha / q}-1\right) x^{*} C x \geqslant\left(\gamma^{\alpha}-1\right) x^{*} A^{r+e_{x}}
$$

where $\gamma=\lambda_{1} / \lambda_{k}$.

In a manner similar to the proof of Theorem 1, Lemma 2 yields the following:

Theorem 4. Let $r, s, p, q, \alpha, \gamma, A$ and $C$ be as defined in Lemma 2 and let $w_{j}, x_{j}, j=1, \ldots, m$ be defined as in Theorem 1. Then

$$
\begin{gathered}
\lambda_{k}^{-\alpha / q}\left(\gamma^{\alpha / p}-1\right) \sum_{j=1}^{m} w_{j} x_{j}^{*} A^{s p} x_{j}+\lambda_{1}^{\alpha / p}\left(\gamma^{\alpha / q}-1\right) \sum_{j=1}^{m} w_{j} x_{j}^{*} C x_{j} \geqslant \\
\left(\gamma^{\alpha}-1\right) \sum_{j=1}^{m} w_{j} x_{j}^{*} A^{r+d} x_{j} .
\end{gathered}
$$


Analogous to the proof of Theorem 5 in [3], we can establish the following:

Theorem 5. Let $r, s, p, q, A, C, \alpha, w_{j}, x_{j}, j=1, \ldots, m$ be defined as in Theorem 4. If $\sum_{j=1}^{m} w_{j} x_{j} C x_{j}>0$, then

$$
\sum_{j=1}^{m} w_{j} x_{j}^{*} A^{r+o} x_{j} \leqslant K\left(\sum_{j=1}^{m} w_{j} x_{j}^{*} A^{s p} x_{j}\right)^{1 / p}\left(\sum_{j=1}^{m} w_{j} x_{j}^{*} C x_{j}\right)^{1 / q}
$$

where

$$
K=p^{1 / p}|q|^{1 / q} \gamma^{\alpha /(p q)}\left(\gamma^{\alpha / p}-1\right)^{1 / p}\left(1-\gamma^{\alpha / q}\right)^{1 / q} /\left(\gamma^{\alpha}-1\right) .
$$

Since $A^{(r q)}$ satisfies the conditions for $C$ in Lemma 2, (19), (20) and (21) give

$$
\begin{gathered}
\lambda_{k}^{-\alpha / q}\left(\gamma^{\alpha / p}-1\right) x^{*} A^{s p} x+\lambda_{1}^{\alpha / p}\left(\gamma^{\alpha / q}-1\right) x^{*} A^{(r q)} x \\
\geqslant\left(\gamma^{\alpha}-1\right) x^{*} A^{r+s} x ; \\
\lambda_{k}^{-\alpha / q}\left(\gamma^{\alpha / p}-1\right) \sum_{j=1}^{m} w_{j} x_{j}^{*} A^{s p} x_{j}+\lambda_{1}^{\alpha / p}\left(\gamma^{\alpha / q}-1\right) \sum_{j=1}^{m} w_{j} x_{j}^{*} A^{(r q)} x_{j} \\
\geqslant\left(\gamma^{\alpha}-1\right) \sum_{j=1}^{m} w_{j} x_{j}^{*} A^{r+s} x_{j} ; \\
\sum_{j=1}^{m} w_{j} x_{j}^{*} A^{r+s} x_{j} \leqslant K\left(\sum_{j=1}^{m} w_{j} x_{j}^{*} A^{s p} x_{j}\right)^{1 / p}\left(\sum_{j=1}^{m} w_{j} x_{j}^{*} A^{(r q)} x_{j}\right)^{1 / q},
\end{gathered}
$$

where $K$ is given by (22).

\section{REFERENCES}

[1] K. Fan, 'Some matrix inequalities', Abh. Math. Sem. Univ. Hamburg 29314 (1966), 185-196.

[2] J.Z. Hearon, 'A generalized matrix version of Rennie's inequality', J. Res. Nat. Bur. Standard (Math. and Math. Phys.) 71B (1967), 61-64.

[3] B. Mond and J.E. Pečarić, 'Inequalities involving powers of generalized inverses', Linear Algebra Appl. (to appear).

Department of Mathematics

La Trobe University

Bundoora Vic 3083

Australia
Faculty of Textile Technology

University of Zagreb

Zagreb

Croatia

and

Department of Mathematics

La Trobe University

Bundoora Vic $\mathbf{3 0 8 3}$

Australia 